\title{
Early experience of inpatient teledermatology in Singapore during COVID-19
}

\begin{abstract}
Dear Editor,
With the COVID-19 pandemic disrupting healthcare systems worldwide, telemedicine has been advocated and adopted globally to meet ongoing challenges of delivering timely medical care, rational allocation of resources, and minimising exposure to patients, healthcare workers and contacts.
\end{abstract}

Since the late 1990s, teledermatology has been utilised to triage, diagnose, monitor and treat skin conditions. ${ }^{1}$ The accessibility and efficiency of dermatology care in underserved communities have also improved with teledermatology. ${ }^{2,3}$ While the utility and cost-effectiveness of teledermatology in ambulatory care have been demonstrated, its role in inpatient dermatological care is less defined. ${ }^{2,4}$ Nonetheless, a small prospective study proposed by Gabel et al. showed that teledermatology in the inpatient setting might be an acceptable option for diagnosis, evaluation and management. ${ }^{5}$

Singapore was one of the first countries to report imported COVID-19 cases in early 2020, with subsequent government public health measures to contain the spread. ${ }^{6}$ Many restructured hospitals reorganised inpatient care, segregated resources, including reserving negative pressure rooms for suspected COVID-19 patients. ${ }^{7}$ Patients with respiratory symptoms or signs of pneumonia were admitted to specific isolation wards called "Acute Respiratory Infection (ARI)" for COVID-19 testing. They were transferred to a general ward bed once tested negative, whereas positive cases remain in isolation.

With this change in care delivery, the department of dermatology undertook a pilot project to evaluate the effectiveness of inpatient store-and-forward teledermatology by comparing the level of diagnostic concordance with face-to-face bedside consultations.

This prospective study included all formal inpatient referrals to dermatology service for patients from isolation wards at the Singapore General Hospital (SGH) from 1 July to 1 October 2020. The referring physician sent photographs using available cameras (including phone cameras) to the inpatient dermatology resident via a secure messaging platform called TigerConnect. TigerConnect is compliant with the US Health Insurance Portability and Accountability Act 1996. No strict photography criteria were imposed, and referring physicians were given the liberty to send any number of photographs deemed appropriate to demonstrate the extent and morphology of the skin rash. The total number of pictures sent in a store-and-forward format was documented. Close-up photographsdefined as photographs illustrating morphology-were also recorded.

The duty inpatient dermatology specialist reviewed the clinical history and submitted photographs from the primary physician, and provided a preliminary reply that is documented electronically on the same day of referral. The preliminary reply included the diagnosis, recommended investigations, and treatment. Patients who were subsequently proven negative for COVID-19 were transferred to the general ward, where the consultant dermatologist would review the patient again by the bedside. The final clinical dermatological diagnosis was then recorded and compared to the preliminary teledermatology assessment.

In the event that the patient remained in the isolation ward due to COVID-19 swab positivity, continuation of care, or insufficient general ward bed resources, patients were discharged with an early review in the dermatology clinic instead.

The primary endpoint was the degree of agreement between teledermatology diagnosis and face-to-face bedside diagnosis. In cases with diagnostic discordance, reasons for failure were categorised into technical (e.g. photography quality), patient, or physician factor.

From 1 July to 1 October 2020, 76 patients from isolation wards in SGH were referred to the inpatient dermatology service. Eleven patients were excluded due to discharge or death prior to physical review. The main diagnostic categories were eczema $(\mathrm{n}=18,27.7 \%)$ immunobullous disorders $(\mathrm{n}=11,16.9 \%)$, cutaneous adverse drug reactions $(\mathrm{n}=8,12.4 \%)$, infections $(\mathrm{n}=11,16.9 \%)$, connective tissue disorders/vasculitis $(n=4,6.2 \%)$, urticaria $(n=5,7.7 \%)$ and skin tumours $(n=2,3.1 \%)$. There was 1 COVID-19-related vesicular eruption.

When comparing store-and-forward teledermatology and bedside diagnoses, the diagnostic concordance was $58 / 65(89.2 \%)$. Seven cases resulted in a change of diagnosis, including connective tissue disorders such as subacute cutaneous lupus erythematosus $(n=1)$, immunobullous disorders $(n=1)$, skin tumours $(n=1)$, psoriasis $(n=2)$, infections including dengue rash $(n=1)$, and urticaria $(\mathrm{n}=1)$. 
Table 1. Cases with a change in diagnosis

\begin{tabular}{|c|c|c|c|c|c|}
\hline Case & Teledermatology diagnosis & Physical review diagnosis & $\begin{array}{l}\text { Days } \\
\text { between } \\
\text { review }\end{array}$ & $\begin{array}{l}\text { Reviewed in } \\
\text { GW vs clinic }\end{array}$ & $\begin{array}{l}\text { Possible reason for the change in } \\
\text { diagnosis }\end{array}$ \\
\hline 2 & Acute eczema & $\begin{array}{l}\text { Autoimmune blistering } \\
\text { disease }\end{array}$ & 1 & GW & $\begin{array}{l}\text { Missing information (diagnostic } \\
\text { lesion not captured) }\end{array}$ \\
\hline 3 & $\begin{array}{c}\text { Nodules for investigation } \\
\text { unable to appreciate } \\
\text { pathology - unclear }\end{array}$ & Urticaria & 2 & GW & $\begin{array}{c}\text { Technical (poor image quality, } \\
\text { diagnostic lesion not captured) } \\
\text { Clinical evolution }\end{array}$ \\
\hline 4 & $\begin{array}{l}\text { Left forearm plaque } \\
\text { TRO SCC }\end{array}$ & $\begin{array}{l}\text { Left forearm haematoma } \\
\text { with possible overlying } \\
\text { seborrheic keratosis }\end{array}$ & 2 & GW & $\begin{array}{l}\text { Technical (poor image quality) } \\
\text { Missing information (inadequate } \\
\text { anatomical sites shown) }\end{array}$ \\
\hline 5 & Asteatotic eczema TRO tinea & Partially treated psoriasis & 1 & GW & Technical (poor image quality) \\
\hline 6 & $\begin{array}{l}\text { Acral blistering dermatosis, } \\
\text { papulosquamous eruption, DDx } \\
\text { pityriasis rosea, sarcoidosis }\end{array}$ & Psoriasis & 24 & Clinic & $\begin{array}{l}\text { Missing information (inadequate } \\
\text { anatomical sites shown) }\end{array}$ \\
\hline 7 & Possible SDRIFE & $\begin{array}{l}\text { Dengue rash, pregnancy } \\
\text { chloasma/lentigines }\end{array}$ & 14 & Clinic & $\begin{array}{l}\text { Missing information (inadequate } \\
\text { anatomical sites shown) }\end{array}$ \\
\hline
\end{tabular}

DDx: differential diagnosis; EN: erythema nodosum; GW: general ward; SCC: squamous cell carcinoma; SDRIFE: symmetrical drug-related intertriginous and flexural exanthema; TRO: to rule out

This pilot study demonstrated that inpatient store-andforward teledermatology has a diagnostic concordance of $89.2 \%$ compared to traditional bedside consultations. Our findings are consistent with the current literature, which has validated store-and-forward teledermatology as an effective care delivery model for inpatient dermatology. ${ }^{5}$

The limitations of this study need to be acknowledged. Our study population was restricted to acute respiratory illness/suspected COVID-19 cases. This might influence the generalisability to the entire inpatient population. The concordance of teledermatology may be influenced by the non-standardised photographic equipment, technique, environment, and protocol, which may result in variability in both the number and quality of clinical photographs. To reduce diagnostic variability bias, the same consultant dermatologist reviewed the pictures and the patient physically. In addition, 4 specialist dermatologists took part in this study, and the variability in the diagnostic concordance was similar, with individual concordance ranging from $85.7 \%$ to $93.3 \%$.

Although the level of discordance was low, possible contributing factors included technical issues such as poor image quality and inadequate photographic information (inadequate lesions or sites were taken) and patient/clinical factors. For example, missing photos of blisters resulted in the misdiagnosis of a nonimmunobullous condition. Unclear photographs due to phone camera storage in the plastic biohazard bag often used as a protective measure during COVID-19, poor lighting, and variable photographic quality settings in TigerConnect were also identified as reasons for difficult assessment.

Unfortunately, missed diagnosis often involved all 3 factors. Patients were reported to be uncooperative, with difficult-to-reach locations such as the buttock cited as reasons for the lack of quality photographs. Non-dermatology-trained physicians may also not recognise the importance of photographic clarity and resolution during the forwarding of images. These challenges can be mitigated by standardisation of the camera, with photos taken under adequate lighting and at least 1 photo demonstrating the area of interest in the setting of a localised rash while ensuring the distribution of rash is included for patients with generalised rash. Close-up imaging is vital in determining morphology. Imaging of associated sites, such as oral mucositis, is also recommended.

While store-and-forward teledermatology has been shown in our study to be an effective model of care, its impact on education and doctor-patient engagement remains unclear. Nonetheless, in this 
ongoing COVID-19 pandemic, there will be continued stress on medical systems with the need to protect patients and healthcare workers. Store-and-forward teledermatology would be a useful care model with a high level of agreement in diagnosis.

\section{REFERENCES}

1. Trettel A, Eissing L, Augustin M. Telemedicine in dermatology: findings and experiences worldwide - a systematic literature review. J Eur Acad Dermatol Venereol 2018;32:215-24.

2. Wang RH, Barbieri JS, Nguyen HP, et al. Clinical effectiveness and cost-effectiveness of teledermatology: Where are we now, and what are the barriers to adoption? J Am Acad Dermatol 2020; 83:299-307.

3. Zakaria A, Maurer T, Su G, et al. Impact of teledermatology on the accessibility and efficiency of dermatology care in an urban safety-net hospital: A pre-post analysis. J Am Acad Dermatol 2019;81:1446-52.

4. Zakaria A, Miclau TA, Maurer T, et al. Cost Minimization Analysis of a Teledermatology Triage System in a Managed Care Setting. JAMA Dermatol 2021;157:52-8.
5. Gabel CK, Nguyen E, Karmouta R, et al. Use of teledermatology by dermatology hospitalists is effective in the diagnosis and management of inpatient disease. J Am Acad Dermatol 2021; $84: 1547-53$.

6. Chen JI, Yap JC, Hsu LY, et al. COVID-19 and Singapore: From Early Response to Circuit Breaker. Ann Acad Med Singap 2020; 49:561-72.

7. Tan THY, Toh MPHS, Vasoo S, et al. Coronavirus Disease 2019 (COVID-19): The Singapore Experience. A Review of the First Eight Months. Ann Acad Med Singap 2020;49:764-78.

Wen Hao $\underline{\operatorname{Tan}}^{1}{ }^{M R C P}$, Chee Hoou Loh ${ }^{1}{ }_{M R C P}$, $\mathrm{Zi}$ Teng $\underline{\mathrm{Chai}}{ }^{1}{ }_{M R C P}$, Dawn AQ $\underline{\mathrm{Oh}}{ }^{1}{ }_{M R C P}$, Choon Chiat $\underline{\mathrm{Oh}}{ }^{1}{ }_{M R C P}$, Yi Wei $\underline{\mathrm{Yeo}}{ }^{1}{ }_{M R C P}$, Karen JL Choo ${ }^{1}{ }_{M R C P}$, Haur Yueh Lee ${ }^{1}{ }_{M R C P}$

${ }^{1}$ Department of Dermatology, Singapore General Hospital, Singapore

Correspondence: A/Prof Haur Yueh Lee, Department of Dermatology, Singapore General Hospital, 20 College Road, Singapore 168856. Email: lee.haur.yueh@singhealth.com.sg 